\title{
Multi-Layer RF Tissue Phantoms for Mimicking a Human Core
}

\author{
Quenton Bonds \\ Microwave Instruments and Technology Branch \\ NASA GSFC \\ Greenbelt MD, USA \\ quenton.bonds@nasa.gov
}

\begin{abstract}
This work presents the design recipe, fabrication process and characterization of tissue-simulating materials, configured as a physical model to mimic the electrical and some physical properties of an abdominal cavity. The complete threelayer design is called the human core model (HCM) see Fig. 1. To our knowledge, presented is the first hybrid skin-muscle phantom developed to mimic the electrical properties of the intervening tissue layers of an abdominal cavity within the frequency band of $1 \mathrm{GHz}-2 \mathrm{GHz}$, a band of interest for human body sensing due to its deep detection depth.
\end{abstract}

Keywords - RF Tissue Phantoms, Tissue Mimicking Materials, Multi-Layer RF Tissue Phantoms, Human Tissue Characterization

\section{InTROdUCTION (HEADING 1)}

Revolutionary advances in wearable sensing technologies with non-invasive monitoring capabilities are driving research and development of materials capable of mimicking the electrical properties of the human tissue and organs. The body is a complex machine, consisting of skin, muscle blood and fatty tissue, with dissimilar electrical and physical properties. As a result, each tissue layer will affect the performance of the sensor. Therefore, discrete phantoms, which model only one tissue layer at a time, do not provide the best replica of the body. For measurement and testing purposes, desired are versatile multifaceted phantom models capable of mimicking the physical and electrical properties of the tissue volume

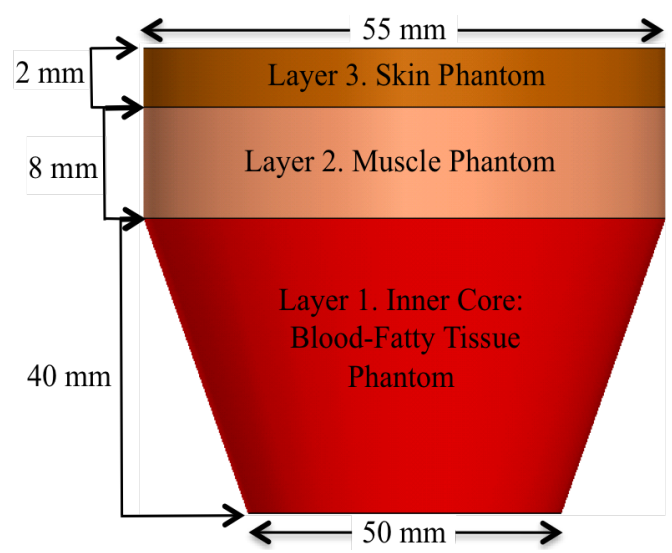

Figure 1. The Human Core Model

${ }^{1}$ Oil Center Research, 616 W. Pont Des Mouton Road, Lafayette LA 70507)

${ }^{2}$ NASA GSFC Nationals Society of Black Engineers (NSBE) Travel Sponsor
Thomas Weller

\author{
Department of Electrical Engineering, RF Microsystems \\ The University of South Florida \\ Tampa FL, USA \\ weller@usf.edu
}

across the sensing depth. Phantom models that replicate various clinical diagnostic points of the body are of particular interest since the performance of the sensor can be improved by designing it for optimum functionality at clinical diagnostic point of interest also referred to as the areas of diagnosis. Thus, the phantoms presented herein mimic the physical properties of human tissue, as well as the electrical properties. The applications of these phantoms models are limitless, biomedical telemetry devices, non-contact wireless sensors and wearable devices. Layered configurations of these phantoms can be used to model virtually any clinical diagnostic points in the body, however in this work, we've modeled a human core, as the broader impact of this work is a close-proximity measurement of core body temperature [1]

\section{The Human CORE Model (HCM)}

\section{A. Motivation}

The HCM was developed to provide a more concise electromagnetic model of an abdominal cavity than the more commonly used single layer phantoms. Such phantoms are usually developed using simple solid, semi-solid (gels), or liquid solutions [2], [3]. Previous studies have proven that "dielectric layering greatly influences" the radiometric measurement, therefore single layer phantoms cannot accurately mimic the emissive properties of layered volumes human tissue [1] \& [4]. This loss in accuracy, could create considerable measurement errors, since microwave radiometers detect very low brightness temperature (TB) emissions which are dependent on the electrical properties of the tissue.

Liquid phantoms, usually comprised of saline or a water bolus, have a dielectric constant similar to water $(\sim 78)$ at room temperature and $1.4 \mathrm{GHz}$, whereas human muscle has a dielectric constant of $\sim 54$, skin $\sim 44$, and fat $\sim 10$. As a result the detectable emissions from liquid phantoms will differ from those of human tissue.

Semisolids or gel phantoms have been developed which have similar electrical characteristics to that of skin and muscle tissue, however they are not as durable as solid phantoms. Though semisolids provide a more accurate representation of the body's electrical characteristics across the surface of the TUI, some disadvantages exist when modeling three dimensional volumes of the body's clinical diagnostic regions. For instance thin tissue layers $(<2 \mathrm{~mm})$ such as skin are difficult to develop and manage. Moreover, the versatility of the test bed is reduced, since less dense semisolids may not 


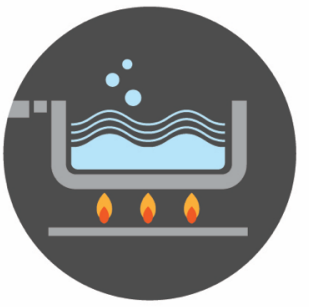

Heat water to boiling point.

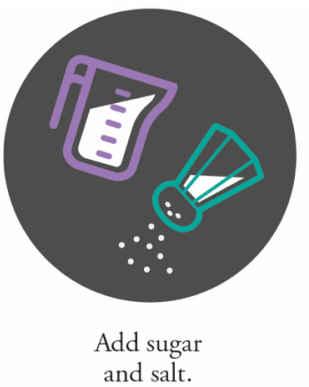

and salt.

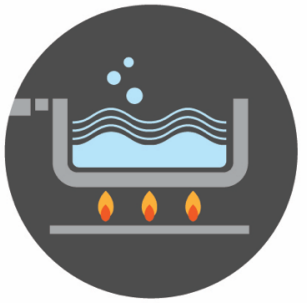

Continue to boil the solution until the compounds are dissolved.

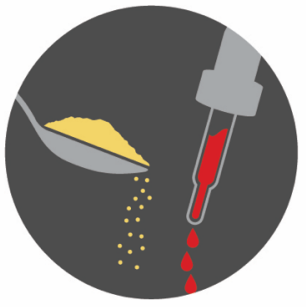

Add HEC and optional red food coloring.

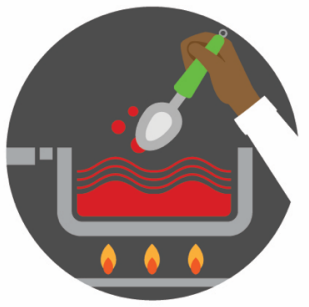

Reduce heat, and continue stirring until the compound is dissolved.

\section{The sample is now prepared for characterization.}

Figure 2. Development process for the blood-fatty tissue phantom.

hold the form factor of the tissue volume, especially at elevated temperatures indicative to that of heat related disorders.

Until this work, no known solid skin-muscle phantoms have been developed with electrical properties analogous to human tissue within the $1-2 \mathrm{GHz}$ frequency band. There are some single layer phantoms (muscle), which employ an outer shell or cast to hold the form of the TUI. However, the electrical characteristics of the casts, usually plastics, are distinctly dissimilar at the air - skin interface. This interface is the first and therefore critical boundary for extracting subsurface data from the TUI, due to sizable reflections, which occur at that boundary which lead to radiometric signal loss. [3] presents a solid 2/3rd muscle phantom which is the currently best available technology for mimicking a human torso. Yet again, this 33\% difference in the dielectric constant may present significant measurement errors when extracting deep-seated tissue temperatures using microwave radiometers.

\section{B. Blood-Fatty Tissue Phantom}

Herein blood-fatty tissue was simulated using a mixture of hydroxethylcellulose (HEC), salt, sugar and water, the most prominent compounds in human blood as well as fatty (cellulose, salt, water) tissue (Table 1) [5]. To model blood and fatty tissue inside the stomach, a weighted average of the dielectric properties of the tissue is applied, assuming $12 \%$ body fat and $88 \%$ blood, which results in a dielectric constant of 53 , and an impedance of $50 \Omega$ at fc. Figure 2 outlines the

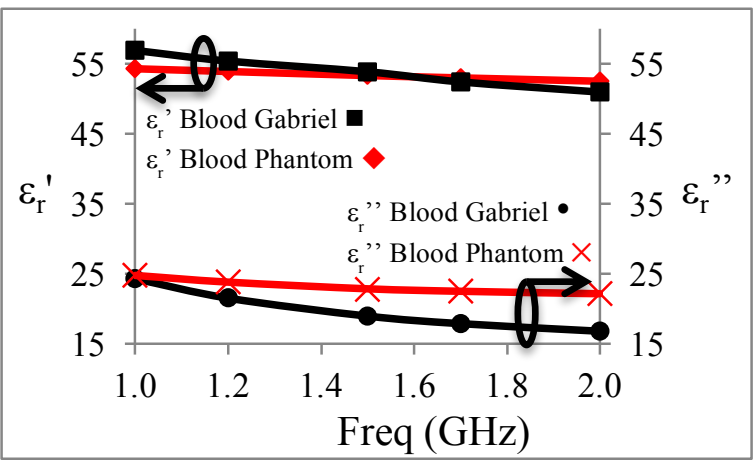

Figure 3. Comparison of the real $\left(\varepsilon_{r}^{\prime}\right)$ and imaginary $\left(\varepsilon_{r}^{\prime \prime}\right)$ dielectric constant $\left(\varepsilon_{\mathrm{r}}\right)$ of the blood-fatty tissue phantom to the Gabriel model.

\begin{tabular}{|c|c|}
\hline Ingredients & $\begin{array}{c}\text { \% By } \\
\text { Weight }\end{array}$ \\
\hline Water & 56 \\
\hline Sugar & 0.76 \\
\hline Hydroxylethylcellulose (HEC) & 41.76 \\
\hline Salt & 1.21 \\
\hline Bactericide & 0.27 \\
\hline
\end{tabular}

Table 1. Recipe for blood-fatty tissue phantom.

development process, and the recipe is provided in Table 1. As illustrated in Figure 3 and Figure 4, the blood phantom has a dielectric constant of 54 and impedance of $51 \Omega$ at $f_{c}$ which is equivalent to the findings of Gabriel [5] and others in the literature. Here, it is important to note that since this phantom is a liquid, it can be easily heated and cooled to mimic physiological characteristics of the human body.

\section{Hybrid Skin-Muscle Tissue Phantom}

Presented is the first hybrid skin-muscle phantom in a solidified composite form. As described in the introduction, the majority of the current tissue phantoms are discrete materials, usually liquids, whereas the human body is

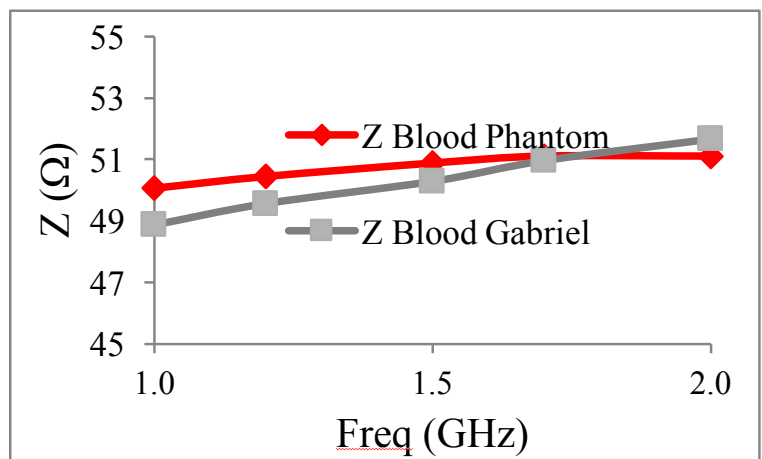

Figure 4. Comparison of the blood-fatty tissue phantom impedance $(Z)$ to the Gabriel model. 


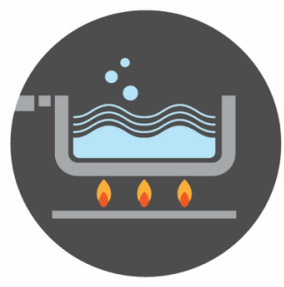

Heat water to boiling point.

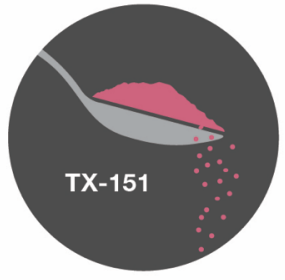

Add TX-151.

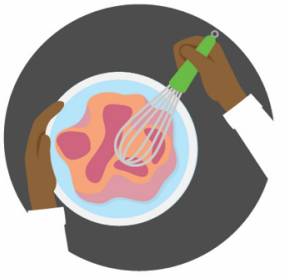

Knead the solution until "all" TX-151 particles are shaped into a single solid form.

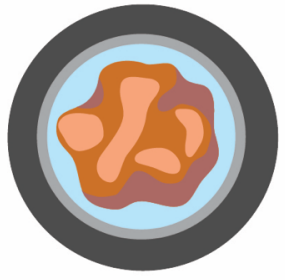

Let the phantom dry until a thin white film covers the surface of the phantom.

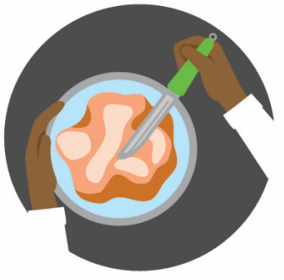

Using a sharp object, scrape off a thin (skin) layer on one face.

The sample is now prepared for characterization.

Figure 5. Development process for the hybrid skin-muscle phantom.

compromised of layered volumes of interconnected solids as well as liquids. Composite or hybrid phantoms provide a more accurate model of the body's clinical diagnostic points, which is important in improving the precision of the measurement test bed, as it is very difficult to model such a complex biological systems as the human body. Solid phantoms can be shaped into three-dimensional volumes of human tissue without the support of casts or containers which may alter the relative dielectric, and or impedance profile of the phantom.

The skin-muscle phantom is developed using a simple mixture of $44 \%$ water and $56 \%$ TX-151, provided by the Oil Center Research1. The process is presented in Figure 5, and the recipe in Table 2. The surface of the hybrid phantom mimics damp skin, to account for perspiration in conditions of extreme heat and or condensation deposits in cold environments. The skin layer has non-uniform thickness, rangi

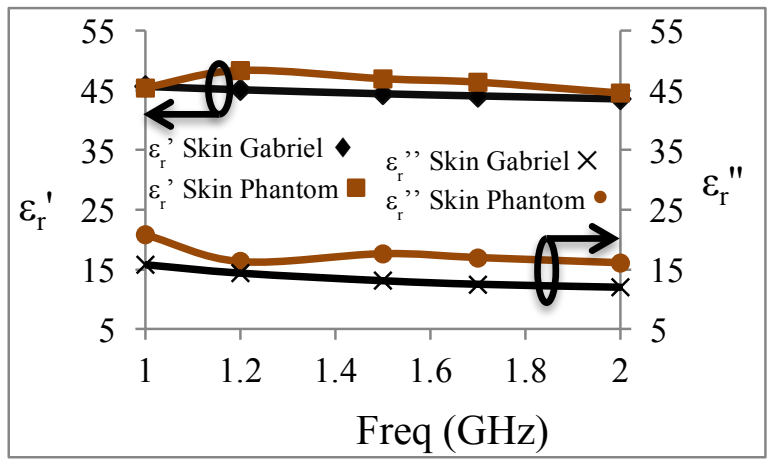

Figure 6. Comparison of the real $\left(\varepsilon_{\mathrm{r}}^{\prime}\right)$ and imaginary $\left(\varepsilon_{\mathrm{r}}^{\prime \prime}\right)$ dielectric constant $\left(\varepsilon_{\mathrm{r}}\right)$ of the skin tissue phantom to the Gabriel model.

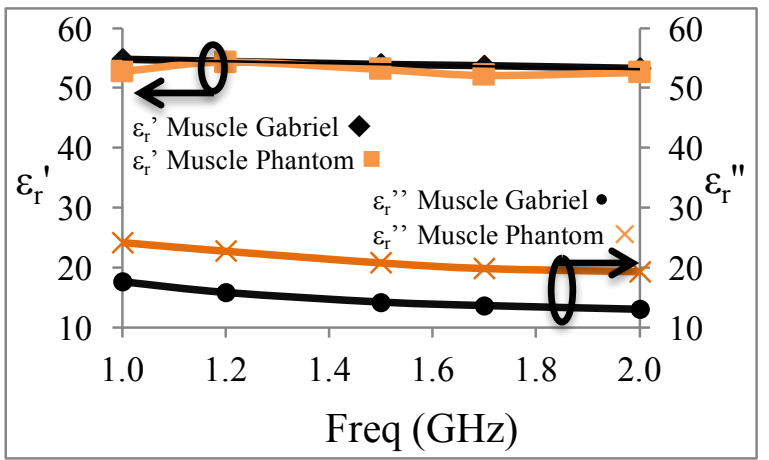

Figure 8 Comparison of the real $\left(\varepsilon_{\mathrm{r}}^{\prime}\right)$ and imaginary $\left(\varepsilon_{\mathrm{r}}^{\prime \prime}\right)$ dielectric constant $\left(\varepsilon_{\mathrm{r}}\right)$ of the muscle tissue phantom to the Gabriel model.

\begin{tabular}{|c|c|}
\hline Ingredients & $\begin{array}{c}\text { \% By } \\
\text { Weight }\end{array}$ \\
\hline Water & 60 \\
\hline TX-151 & 40 \\
\hline
\end{tabular}

Table 2. Recipe for hybrid skin-muscle phantom.

ng from $1 \mathrm{~mm}-2 \mathrm{~mm}$, which is comparable to the combined thickness of the dermis, epidermis, and hypodermis. The muscle layer is located immediately below the skin layer, and also has a non-uniform thickness of $7 \mathrm{~mm}$ to $8 \mathrm{~mm}$. The electrical characteristics of the skin and muscle layers are presented in Figure 6 through Figure 10. When preserved in an airtight enclosure, the electrical and physical characteristics of the phantom will remain relatively stable for at least 6 months.

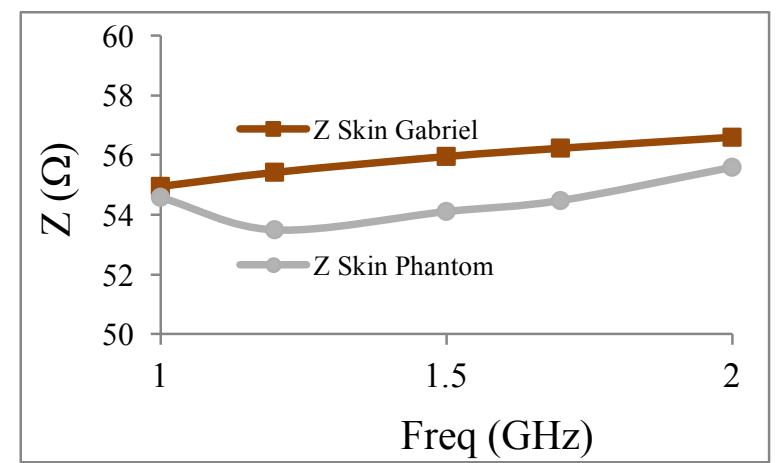

Figure 7. Comparison of the skin tissue phantom impedance $(\mathrm{Z})$ to the Gabriel model.

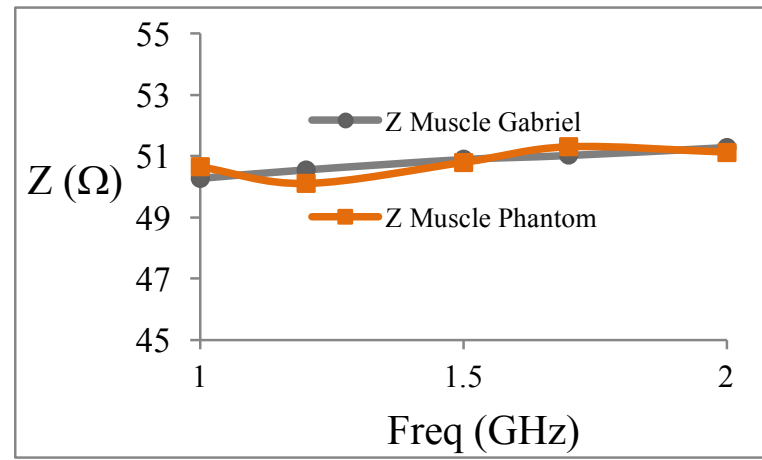

Figure 9. Comparison of the muscle tissue phantom impedance $(\mathrm{Z})$ to the Gabriel model. 

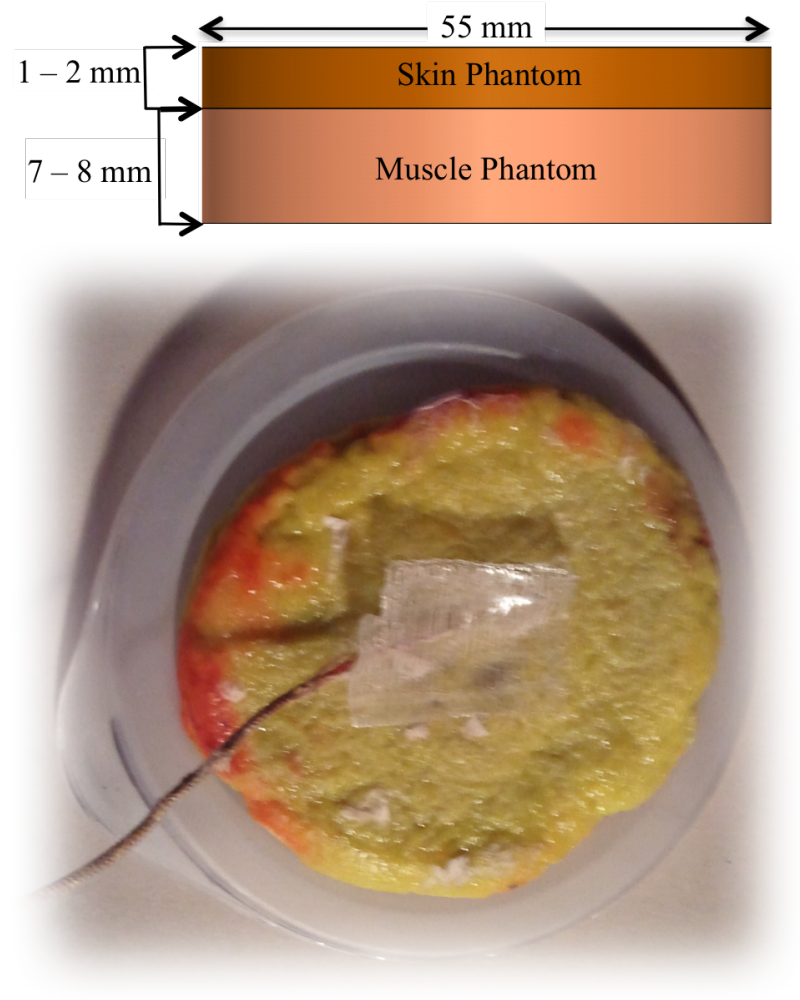

Figure 10. Hybrid Skin-Muscle Phantom:

Bottom - actual phantom; Top - side view model of intervening layers

\section{The Human Core Model (HCM)}

The HCM is a durable phantom, designed to mimic the region of diagnosis for core body temperature monitoring, using a layered configuration of the phantoms previously presented in section II. Layers 1 and 2 of the HCM make up the hybrid skin-muscle phantom, comprised of a composite material developed using water and TX-151. The skin layer has a non-uniform thickness, ranging from $1 \mathrm{~mm}-2 \mathrm{~mm}$, which is comparable to the combined thickness of the dermis, epidermis, and hypodermis. The muscle layer is located immediately below the skin layer, and also has a non-uniform thickness of $7 \mathrm{~mm}$ to $8 \mathrm{~mm}$. Core body temperature is essentially based on the temperature of the circulating blood through the cranial, thoracic and abdominal cavities [6]. Therefore Layer 3, the inner core, is a liquid volume $\sim 40 \mathrm{~mm}$ deep which mimics blood and fatty tissue inside the stomach. The inner core is located inside of a plastic container which with housing to secure the skin-muscle phantom approximately $1 \mathrm{~mm}$ in thickness. Since the radiometer detects thermal emissions across the depth of the tissue, the effect of the container is negligible, being that it's a thin, highly emissive material, located beneath the critical air-skin interface. Herein, extreme body temperature changes are simulated by varying the temperature of the inner core to temperatures representative of heat related disorders.

\section{RESULTS AND CONCLUSION}

The phantoms were characterized with respect to complex dielectric constant using the Agilent $85070 \mathrm{E}$ dielectric probe kit, and the results compared to the Gabriel model, which is used as the standard for human tissue characterization [7]. Since the tissue impedance is an important parameter for characterizing on-body sensors and near field antenna performance, the impedance of the phantoms were also calculated and presented in the analysis. Due to the strong correlation with the Gabriel model, with respect to impedance, real and imaginary dielectric, our findings confirm with great confidence that the methods presented herein are more than sufficient in developing skin-muscle and blood phantoms with electrical as well as physical properties analogous to the human body. Our results are nearly identical to the measurements of the Gabriel model, which vary by temperature and from person to person. Although we have developed this model for a human core, these phantoms can be shaped to just about any area of the human body.

\section{REFERENCES}

[1] Q. Bonds, J. Gerig, T. Weller, and B. Roeder, "Towards Core Body Temperature Measurement via Close Proximity Radiometric Sensing," IEEE Sensors Journal, Special Issue on Non-Invasive Physiological Monitoring, P. 519-526, February 2012

[2] T. Yilmaz, T. Karacolak, and E. Topsakal, "Characterization of Skin Mimicking Gels for Implantable Antennas Operating at ISM Band (2.4 $\mathrm{GHz}$ - 2.48 GHz)," National Radio Science Meeting URSI, 2008

[3] K. Ito, "Numerical and Experimental Human Body Phantoms," IEEE IET Seminar on Antennas and Propagation for Body-Centric Wireless Communications, 2007

[4] M.S. Hawley, J. Conway, A.P. Anderson, and P.A. Cudd, "The influence of tissue layering on microwave thermographic measurements," International Journal of Hyperthermia, vol. 4, no. 4, pp. 427-435, July 1988

[5] Australian Communication Authority, "Radiocommunications (Electromagnetic Radiation Human Exposure) Standard 2003," Radio Communications Act, 1992 Section 162, June 2003

[6] C. L. Lim, C. Bryn, and J.K.W. Lee, "Human thermoregulation and measurement of body temperature in exercise and clinical settings," Annals Academy of Medicine, vol. 4, pp. 347-353, April 2008

[7] C. Gabriel, and S. Gabriel, "Compilation of the Dielectric Properties of Body Tissues at RF and Microwave Frequencies," Brooks AFB report number AL/OE-TR-1996-0037, 1996 